\title{
Proteomic studies in breast cancer (Review)
}

\author{
XIAN-JU QIN ${ }^{1}$ and BRUCE X. LING $^{2}$ \\ ${ }^{1}$ Department of General Surgery, Shanghai Eighth People's Hospital, Shanghai 200235, P.R. China; \\ ${ }^{2}$ Department of Surgery, Stanford University School of Medicine, Stanford University, Stanford, CA 94305, USA
}

Received October 31, 2011; Accepted January 13, 2012

DOI: $10.3892 / \mathrm{ol} .2012 .573$

\begin{abstract}
Breast cancer is one of the most common types of invasive cancer in females worldwide. Despite major advances in early cancer detection and emerging therapeutic strategies, further improvement has to be achieved for precise diagnosis to reduce the chance of metastasis and relapses. Recent proteomic technologies have offered a promising opportunity for the identification of new breast cancer biomarkers. Matrix-assisted laser desorption/ionization, time-of-flight mass spectrometry (MALDI-TOF MS) and the derived surface-enhanced laser desorption/ionization mass spectrometry (SELDI-TOF MS) enable the development of high-throughput proteome analysis based on comprehensive reliable biomarkers. In this review, we examined proteomic technologies and their applications, and provided focus on the proteomics-based profiling analyses of tumor tissues/cells in order to identify and confirm novel biomarkers of breast cancer.
\end{abstract}

\section{Contents}

1. Introduction

2. Proteomics technologies

3. Diagnostic marker protein profiling studies

4. Prognostic and predictive marker protein profiling studies

5. Conclusions

\section{Introduction}

The human genome contains approximately 35,000 genes and has the ability to encode up to 35,000 corresponding proteins. The occurrence of alternative RNA splicing and post-translational modifications (PTM), including phosphorylations,

Correspondence to: Dr Xian-Ju Qin, Department of General Surgery, Shanghai Eighth People's Hospital, 8 Caobao Road, Shanghai 200235, P.R. China

E-mail: qinxj@hotmail.com

Key words: breast cancer, proteomics, biomarkers, matrix-assisted laser desorption/ionization time-of-flight mass spectrometry, surface-enhanced laser desorption/ionization time-of-flight mass spectrometry acetylations, glycosylations and protein cleavages may increase the expression of proteins to 500,000-1,000,000 (1). Providing the direct link between gene sequence and cell physiology, proteomics is expected to complement genomic analyses to evaluate disease development, prognosis and response to treatment (2).

Breast cancer is one of the most challenging diseases, endangering the health of females worldwide, with the highest incidence found in developed countries. More than one million new cases occur every year, resulting in breast cancer being the leading cause of mortality in females aged 40-59 years. In the United States in 2010, a total of 207,090 new cases of this cancer were estimated to have occurred with an expected mortality of approximately 39,840 women (3). In addition, one-third of patients with initial breast cancer experienced recurrence or metastasis of the disease later (4). A major challenge in breast cancer care is the identification of reliable biomarkers that improve early diagnosis, screening, prediction of outcome, therapeutic response, toxicity and identification of novel target therapies. Proteome studies in breast cancer require tissue samples and biological fluids, including serum, plasma, saliva, nipple aspirate and cerebrospinal fluid $(5,6)$.

Despite the current limitations, proteomics-based biomarker discovery and validation are capable of improving the molecular knowledge of breast cancer and the creation of tissue-based or circulating diagnostic and prognostic clinical utilities (7). In this review, the current techniques applied to breast cancer studies and the most significant research results are summarized.

\section{Proteomics technologies}

The polyacrylamide gel-based platforms, including bidimensional electrophoresis (2-DE) and its variant two-dimensional fluorescence difference gel electrophoresis (2-DE DIGE), consist of tagging two or three protein extracts with various fluorescent molecules (Cy2, Cy3 or $\mathrm{Cy} 5)$ that are subsequently blended and separated on a single gel. In 2-DE, the complex protein samples are separated according to their isoelectric charge and then by their molecular weight (MW) using electrophoresis. The direction of the protein movement at the second phase is perpendicular to the first, in order to provide a spot map of the proteins distributed in the two dimensions. The 2-DE-separated proteins are detected using several pre- or post-electrophoresis staining or fluorescence techniques (8). In the traditional method of $2-\mathrm{DE}$, proteins are separated from 
a complex mixture according to their electrical charge and dimensional differences. The advantage of this method is that large numbers $(3,000-10,000)$ of proteins can be separated visually. The problems of repeatability and standardization associated with this method are overcome with the use of an immobilized $\mathrm{pH}$ gradient (IPG) (9).

Proteomic analyses are differentiated according to whether or not they use mass spectrometry (MS) in the methodology. Non-MS-based approaches require prior knowledge of the proteins that are to be tested; they use specific antibodies and include techniques such as enzyme-linked immunosorbent assay (ELISA), immunohistochemistry (IHC), western blotting and more recently, tissue microarray (TMA) and protein microarray (PMA). By contrast, approaches that use MS do not require previous biological knowledge of the proteins (10). Since 1998, TMA has become the most used proteomic approach in oncology. TMA simultaneously analyzes a new protein marker or a group of 'protein signature' markers in hundreds to millions of cylindrical fragments of clustered tumor samples, collected from original paraffin blocks. TMA associated with IHC allows the trials to be performed using the same technical conditions with promptness and viable costs, making it a powerful tool in investigative pathology (11). This has been employed in the cell lysates of breast cancer to evaluate the activation of Her-2 receptors with its ligand following specific actions of receptor inhibitors (12). However, the analyses are limited to those performed with known antibodies (5).

MS, which defines and measures large numbers of unknown proteins following chemical or physical separation, produces a large number of protein markers in order to define the tumor phenotype. However, substantial sample preparation is required prior to analysis. MS analysis of complex protein samples is performed by determination of the mass/ charge ratio $(\mathrm{m} / \mathrm{z})$ and the number of ions for each $\mathrm{m} / \mathrm{z}$ value of a pressurized gas phase ion mixture (13). A mass spectrometer consists of an ionization source, a mass analyzer and a detector. According to the ionizing source, the most widely used MS techniques for evaluating clinical samples are matrix-assisted laser desorption-ionization time-of-flight mass spectrometry (MALDI-TOF MS), its variant surface-enhanced laser desorption ionization time-of-flight mass spectrometry (SELDI-TOF MS), and electrospray ionization mass spectrometry (ESI MS). For analysis of the mass spectrum, statistical and bioinformatics tools are required. Commercially available applications include Proteome, Quest, Propek, Bamf and Biomarker Wizard (14).

In certain literature, attention has been drawn to two significant points regarding MS analyses. One issue involves the selection process prior to data analysis of the spectra, which has poor quality, a lack of analytical validity and the quality control procedures performed for the relevant analysis. This procedure cuts down noise in the proteomic data, and is reflected as lower variability in diagnostic validity (15). The other issue involves diagnostic validity. The MS protein pattern data are collected from small sample size case groups compared to the large amount of data. However, targets should be selected by definite criteria and with as few variables as possible within the small group.

In addition to the peptide mass fingerprint, another technique entitled tandem MS or MS/MS may be performed.
This method is conducted from a secondary fragmentation of peptides in order to obtain a peptide sequence, that, in turn, is searched for in sequence databases for protein identification (16). In conjunction with MS, several methods may be performed beforehand to separate the proteins extracted from the complex samples. For protein separation, high-performance liquid chromatography (HPLC) and multidimensional protein identification technology (MudPIT) coupled with $\mathrm{MS} / \mathrm{MS}$, enable rapid analysis and identification of the peptide sequences obtained. Recently, MS-based 'off-gel' quantitative proteomics methods have been employed, which have provided a means of increasing the number of proteins identified. A multiplex analysis consisting of up to eight samples can be achieved using isobaric tags for relative and absolute quantitation (iTRAQ) technology. iTRAQ has certain advantages; it provides an opportunity to incorporate internal control samples for normalizing different patient sets from distinct experiments, and combines the peptide signals, increasing the chance of generating quality MS/MS for a greatly definitive protein identification (17). A preliminary study using iTRAQ-2D-LC-MS/MS has compared three low-grade breast cancer tissue samples with various metastatic potentials (primary tumor without metastasis, lymph node metastasis and distant metastasis). It was possible to identify 605 non-redundant proteins, demonstrating the ability of the method to define the differential protein spectrum in relation to disease progression, confirmed using quantitative real-time polymerase chain reaction (qRT-PCR) (18).

\section{Diagnostic marker protein profiling studies}

The goal of mass spectrometry-based protein profiling studies performed for breast cancer is to identify novel diagnostic markers. For genetic breast cancer classifications, the sporadic breast cancer subgroups constitute approximately $90 \%$ of cases and hereditary cases constitute approximately $10 \%$ (19). In addition, breast cancers differentiate according to their mutation conveyance in the BRCA1 and BRCA2 genes. This genotypic classification has been confirmed using DNA microarray with a series of genes also containing the cyclin D1 (CCND1) gene (31). Palacios et al (20) confirmed this molecular classification using a protein level study with 37 protein biomarkers. BRCA2 cancers have been found to be correlated with the cycle regulators, D-type cyclines (D1, D3) and CDK4. The BRCA1 cancers have been found to be correlated with ER/Her-2 negativity, rapid proliferation and widespread basal phenotype (21). By classifying lobular and ductal cancers, the proteins EMP1, DVL1, DDR1, PRKC1 and E-cadherin were identified as biomarkers.

Breast cancers are divided into estrogen receptor (ER)-positive and ER-negative groups. Using cell type and signal pathway protein biomarkers, five molecular subtypes of ER-positive breast cancer have been identified. These molecularly defined subtypes are luminal A, luminal B, basal, HER-2 overexpression and normal (22). Together with the pathological characteristics of breast cancer, 97 biomarker proteins were found, including ER, PR, HER-2, p53, CK5/6, CK8/18, cyclin E, Ki-67, BCL2, cyclin D1 and E-cadherin (23). In the evaluations performed in conjunction with survival periods, 26 proteins were selected for follow-up and the 
5 -year survival period was determined to be $80 \%$. In addition to these proteins, in a study performed on four separate groups, functional protein patterns and survival expectations were classified into 3 different subgroups (A1, A2 and B) (24). The study by these authors revealed over 10 protein markers that may distinguish disease subgroups clinically and biologically more reliably than the prognostic markers that were in use. For cancer diagnosis, protein profiles are obtained from control and patient samples and then compared to detect significant protein patterns unique to each group. Studies have described and compared in situ or infiltrating ductal carcinoma with normal tissues or non-malignant tumors (25). As tissue proteins are likely to reflect the earliest changes caused by the successive genetic mutations that lead to breast cancer, it has been hypothesized that the concentration of potential biomarkers is highest in the tumor and immediate microenvironment. Analysis of tumor tissue lysates using SELDI-TOF MS revealed several peaks that were significantly associated with lymph node status or cancer subtype (26).

The high cell heterogeneity of whole tumor tissue specimens complicates the search for tumor-originating proteins. However, this search can be aided by laser capture microdissection (LCM), which enables the selective capture of a specific subset of cells (27). Captured cells can be mounted directly onto a MALDI target following microdissection, thereby maintaining the spatial conformation for imaging MS. Umar et al (28) detected nine differentially expressed tryptic peptides following an analysis of stromal and tumor cells that were collected from five tissue specimens using LCM. Subsequently, Sanders et al (29) identified ubiquitin and S100-A8 to be decreased in tumor tissue $(n=122)$ compared to normal tissue $(n=167)$, whereas S100-A6 was found to be increased. The use of a split-sample approach allowed successful within-study validation of the three potential markers. Since both ubiquitin and S100-A6 were also found to be decreased in the lysates of breast cancer cell lines following apoptosis induced by chemotherapy (30), these proteins may provide potential insights into the pathogenesis of breast cancer following further investigation.

A step towards the identification of signaling proteins regulated in cancer cells has been provided by the molecular chaperone 14-3-3o. The 14-3-3 proteins form a family comprising seven greatly conserved isoforms with a molecular weight of 25-30 kDa. These proteins are involved in the regulation of the cell cycle machinery at several key points. Additionally, they appear to be associated, directly or indirectly, with signaling proteins including IGF-1 receptor, Raf, MEK kinases and PI3-kinase, although the precise molecular mechanisms of inhibition or activation of these elements are not clear (31). Several studies have reported subregulation of the 14-3-3 $\sigma$ protein in breast cancer, suggesting its role as a tumor suppressor (32). However, the difference in expression found between the normal and tumor tissue was not statistically significant, which contradicts previous evidence that demonstrated that the 14-3-3 $\sigma$ protein was an early detection marker of breast lesions (33).

Recently, Kabbage et al (34) identified the overexpression of the $\alpha$-B-crystallin (Hsp5) and Hsp27 molecules in breast tumor samples. Due to the ability of the chaperones (Hsps) to avoid accumulated stress, unfolding and aggregation of the recently formed protein, its expression has significant implications on cell proliferation. Previous studies revealed that $\alpha$-B-crystallin may be a good target for modulating apoptosis pathways, considering that high levels of these proteins were observed in in situ ductal carcinoma (35). Notably, Moyano et al (36) demonstrated that $\alpha$-B-crystallin appears to be sufficient for cancer transformation. It induces EGF and anchorage-independent growth, increases cell migration and invasion, and constitutively activates the MAPK kinase/ERK (MEK/ERK) pathway; indicating that $\alpha$-B-crystallin is a novel oncoprotein. These data suggest that such chaperones may be significant in the carcinogenesis process and are potential diagnostic markers.

Li et al (37) identified three protein pattern biomarkers for breast cancer; one reducing $(4.3 \mathrm{kDa})$ and two increasing (8.1 and $8.9 \mathrm{kDa}$ ). In subsequent studies, their structural definitions were found to be inter- $\alpha$-tyripsin inhibitor heavy chain H4 (ITIH4), C3a des-arginine-C terminal truncated peptide (C3adesArg $\Delta 8$ ), and C3a des-arginine (C3adesArg), respectively; these have been repeated in independent case groups (38). However, in later studies, the increase in cancer cases of the $8.1 \mathrm{kDa}$ marker was not found to be noteworthy, and the $8.9 \mathrm{kDa}$ marker was reported to have decreased in metastatic recurrences (39). Additionally, the ITIH4 (4.3 kDa) fragment has been found to be increased in cancer cases $(40,41)$. Thus, the value of these three biomarkers in the diagnosis of breast cancer remains controversial. As ITIH4 and the use of markers such as fibrinopeptide A, fibrinogen $\alpha, \mathrm{C} 3 \mathrm{f}, \mathrm{C} 4 \mathrm{a}$, apolipoprotein A-VI, bradykinin, factor VIII and transthyretin reflect the clotting status in the blood of cancer sufferers, they are suggested for diagnostic and classification purposes. The serum and plasma levels of these markers suggest variations specific to the matrix (42).

Hudelist et al (25) used 2-DE and MALDI-TOF in a comparative study of tissue fragments and cells collected using LCM from normal and tumor breast tissues of five patients with breast cancer. Proteins expressed in the manually cut tissues were proteins of high MW and low isoelectric point, mainly the extracellular matrix proteins, but also the fibroblasts and endothelial cells. This feature was absent in LCM-extracted cells, in which proteins of intermediate MW and high isoelectric point prevailed. A total of 32 epithelial proteins were differentially expressed and identified as cytokines, structural proteins, tumor-suppressor genes, signal-transducers or cell-cycle regulators. Among the proteins identified, the Maspin (tumor suppressor protein), DCC (membrane protein), and DSG3 (lipoprotein) were subregulated, indicating their possible role in tumor invasion suppression. HSP27, HER-3 and $\mathrm{CATH}$ were overexpressed in the tumor tissue. Certain overexpressed proteins did not have previous reports of association with breast cancer, including CGG3, which has a potential role in malignant transformation in the breast epithelium and AAAS, also referred to as ALADIN (2).

Pietrowska et al (43) performed a protein expression analysis in cells obtained by LCM of frozen breast tumor sections and normal breast epithelium sections using MALDI MS. Protein and peptide expression in invasive mammary carcinoma versus normal mammary epithelium, and ER-positive versus ER-negative tumors were compared. Biomarker candidates were identified using statistical analysis, and classifiers 
were developed and validated in blinded test sets. Several of the $\mathrm{m} / \mathrm{z}$ features used in the classifiers were identified using liquid chromatography-mass spectrometry (LC-MS/MS) and two were confirmed by IHC. A decrease in ubiquitin and S100-A8 (calgranulin A) was revealed in the tumor tissue $(n=122)$ compared to the normal tissue $(n=167)$, whereas S100-A6 (calcyclin) had an increased expression in the tumor tissue. Therefore, three potential markers were identified.

Using 2-D DIGE and MALDI-TOF MS, Schulz et al (44) characterized protein expression patterns of triple-negative (ER, PR and Her-2) breast cancer compared to Her-2-positive tumors. In the study, protein extraction of the microdissected tumor (pureness $>70 \%$ ) was conducted, and the pooled protein extracts from two groups of frozen tumor tissues (TN and Her-2+) were used for the 2-D DIGE gel performance. The use of this procedure ensured high protein performance. The structural proteins, including cytokeratins, vimetin, fibronectin and L-plastin, glycolytic enzymes, annexin-family (ANXA 1 and 2) and peroxiredoxin-family (PRX1) proteins were identified. IHC and western blot analysis results supported the validity of the results. The identified marker proteins could advance a more detailed characterization of triple-negative breast cancers and may contribute to the development of improved treatment strategies.

Due to improvements in multiple genetic and histological approaches, over the past decade, developments of new breast cancer diagnostics and treatment paradigms have accelerated. Accordingly, a number of distinct genetic subtypes of breast cancer have been defined, with the aim of progressively leading towards more personalized medicine in regard to treatment options. However, there remains a deficiency in the development of molecular diagnostic assays that can be applied for breast cancer detection and used for preclinical decisions (45); for example, the type of cancer-specific biomarker that should be typified by a serum or tissue-derived protein. Progress in this regard is minimal compared to the rapid advancements in genetic and histological assays for breast cancers (46).

\section{Prognostic and predictive marker protein profiling studies}

The aim of prognostic marker protein profiling studies is to classify patients with varying clinical outcomes. The first prognostic gene signature (47) consisted of 70 genes and was demonstrated to identify a group of good-prognosis patients with minimal risk of development of distant metastasis within 5 years in patients undergoing systemic therapy. In a subsequent study, Buyse et al (48) demonstrated that the 70-gene signature was a predictor of outcome independent of the current clinicopathological prognostic markers in a dataset comprising 295 cases. Significantly, in that and subsequent studies (49), it has been repeatedly demonstrated that the 70 -gene signature classifies greater than $95 \%$ of ER-negative cancers as poor prognosis, and that there is a strong correlation between a 70-gene signature-defined poor prognosis and a high histological grade. Subsequent studies have led to the development of various other prognostic signatures, including the 76-gene signature and genomic grade index (50), which were also shown to be independent predictors of outcome.
Bertucci and Goncalves (10) investigated the post-operative sera of 83 high-risk breast cancer patients using SELDI-TOFMS, by constructing a 40 -protein signature that correctly predicted outcome in $83 \%$ of patients. Major components of this signature included haptoglobin $\alpha-1$, complement component $\mathrm{C} 3 \mathrm{a}$, transferrin and apolipoprotein A-I and C-I. These results should be interpreted cautiously, as the number of proteins used for classification is rather high in comparison with the limited study population, indicating possible over-fitting of the data. Moreover, there is a marked association between the haptoglobin phenotype and the recurrent free survival in the sera of 63 high-risk primary breast cancer patients. However, the results have not yet been validated in independent sample sets (51). As the results were not confirmed following validation by haptoglobin phenotyping of a six-fold larger sample set $(n=371)$, utilization of SELDI-TOF MS showed that this likely resulted from a type I error (i.e., false positive).

In a third SELDI-TOF MS study performed in breast cancer tissue $(n=60)$, good prognosis was found to be associated with high levels of ubiquitin and/or low levels of ferritin light chain (52). Notably, ubiquitin has also been found to be differentially expressed in breast cancer by three other studies investigating tissue specimens and cell lines (53). To evaluate the prognostic markers in a SELDI-TOF MS study of tumor tissue from 60 patients with breast cancer, of which 30 patients had no recurrence and the remaining 30 had metastasis, several differentially expressed proteins were identified. Two of the proteins had a strong prognostic value; high levels of ubiquitin and/or low levels of ferritin light chain were associated with good prognosis, as confirmed by IHC and western blot analysis (54). Another SELDI-TOF MS study using LCM, analyzed the tumor tissues of 65 patients with or without axillary compromise and identified two peaks $(4,871$ and $8,596 \mathrm{Da})$ differentially expressed for the conditions; the 8,596 Da peak corresponded to ubiquitin. This peak was under-expressed in breast cancers with axillary lymph node metastasis, indicating that ubiquitin is a good prognostic biomarker (55). Ubiquitin was differentially expressed in two further cell lineage studies (56). Despite these concordant findings, the results have not been confirmed by an analysis of independent sample sets.

In the search for prognostic markers, two studies have investigated the correlation between SELDI-TOF MS protein profiles of tumor tissue lysates and breast cancer cell lines with previously reported breast cancer subtypes (57). Although discrepancies between cells grown in vivo and in vitro exist due to adaptation caused by cell culture conditions, breast cancer cell lines were demonstrated to accurately reflect the genomic, transcriptional and biological heterogeneity found in primary tumors. Within the several differentially expressed protein peaks detected, heat shock protein (HSP) 27 and annexin V were found to be overexpressed in the lysates of the luminal A type tumor tissue (58), while the S100-A9 and a C-terminal truncated form of ubiquitin were found to be differentially expressed between the luminal-like and basal-like cell lines. Subsequent IHC analysis of S100-A9 in tumor specimens of 547 early breast cancer patients confirmed its association with basal subtypes, as well as its value as a poor prognosis indicator (59). However, the prognostic potential of HSP 27 and annexin $\mathrm{V}$ in vivo requires assessment by validation in clinical tissue samples. 
A combination of HPLC and MALDI-MS termed the 'differential peptide display' was used to study peptides generated from 80 invasive ER-positive and ER-negative breast tumors. The results demonstrated the identification of differentially expressed proteins, which were correlated with hormonal status (60). Notably, this analysis revealed a peptide with a $\mathrm{m} / \mathrm{z}$ ratio of 3,108 corresponding to thymosin $\alpha-1$. Prothymosin- $\alpha$ (PTa) was found to differentiate the tumor samples according to their receptor status with the highest specificity. The concentration of thymosin $\alpha-1$ was found to be upregulated in ER-negative cancer samples and downregulated in ER-positive breast cancer samples. In studies performed with the multiple protein patterns, AIB1, MUC1, MUC3, COX2, TERT, EpCAM/TACSTD1, EPIL, crystalline $\alpha-\mathrm{B}$, cytokeratin 5 and 17, and annexin A8 in the basal group, have been linked with poor clinical trend. However, STAT5, BCL2 and GATA3 in the ER-luminal group as well as GATA4, Ki-67 and Her-2 in the Her-2 group, have been linked with a favorable clinical trend $(37,61)$. Hodgkinson (62) reported that a 21 protein pattern defined in a 5 -year metastasis prediction of survival study concluded more favorably than the current standards, and in an independent study, 9 of these proteins were approved.

An accurate prediction of chemo-sensitivity in cancer therapy would enable individualized therapy while avoiding toxic side effects and eliminating the use of ineffective agents. However, protein profiling studies searching for markers for response prediction and treatment monitoring of breast cancer are scarce. The correct prediction of chemo-sensitivity in cancer therapy may cause a reduction in the use of ineffective medication and improve clinical results via protection from side effects. Using studies performed on drug-sensitive and drug-resistant (doxorubicin and paclitaxel) breast cancer cells with SELDI-TOF MS to determine biomarkers that may provide prediction of therapeutic response and follow-up of therapy, a large number of structurally undefined protein peaks have been proposed (63). Maurya et al proposed the use of transferrin fragments, linked with poor clinical progress, for the prediction of paclitaxel resistance. In treatments stimulating apoptosis, a decrease in ubiquitin and S100-A6, and an abnormal expression in breast cancer tissue was found (64). However, the results of these studies have yet to be transferred to clinical samples. Among the limited number of studies performed in vivo in this field, it was determined that kininogen and apolipoprotein A-II decreased in the shock table due to the effect of docetaxel, and the structurally undefined SELDI peak, determined as $2790 \mathrm{~m} / \mathrm{z}$, markedly increased following infusion of paclitaxel (65). However, as yet it has been difficult to define a proteomic predictive marker capable of predicting either the therapeutic response to micrometastatic carcinomas or the side effects caused by the cytotoxic treatment in a patient.

Utilizing fresh breast cancer tissue samples, a number of studies based on proteomics have been conducted; however, the majority of these are based on comparisons between the proteome of normal tissue versus malignant samples, rather than investigations of chemotherapy response. These proteomic studies addressed the technical issues regarding the heterogeneity of breast cancer tissue through the use of LCM (66), the presence of highly-abundant proteins from contaminating blood serum through the use of depletion strategies and also successfully utilized the limited amount of tissue available in pretreatment diagnostic biopsies (67). A search for biomarkers with the ability to predict response to neoadjuvant chemotherapy using proteomic methods in breast cancer tissue has not yet been published. The investigation of chemotherapy response using clinical breast cancer samples is required for further research (62).

ER status has a significant negative predictive value for evaluating the response to anti-estrogen therapy. Sensitivity to the endocrine therapy (SET) index is based on the principle that the expression of genes correlated with ER may greatly improve the prediction response to endocrine treatment compared to ER expression alone. Recently, Loi et al (68) reported promising results focusing on phosphoinositide-3-kinase, catalytic (PIK3CA) gene mutations and the PI3K-AKT-mTOR signaling pathway targeted by PI3K/mTOR (mammalian target of rapamycin) inhibitors. By analysis of gene expression from 1,800 breast cancers, a gene expression signature associated with PIK3CA mutation was developed (PIK3CA-GS). The signature predicted PIK3CA mutations in two independent datasets of breast cancers and was revealed to identify good prognosis in ER-positive patients, the Her-2-negative breast cancer subgroup, and even in highly proliferative tumors. Breast cancer cell lines with high PIK3CA-GS were confirmed to be resistant to rapamycin (69).

Using 2-DE analysis and MALDI-TOF MS, Liu et al (70) demonstrated that cell lines of human cancer which

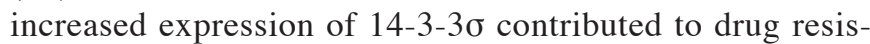
tance, suggesting that such a change is associated with clinical resistance to chemotherapy. In treatments stimulating apoptosis, ubiquitin and S100-A6 decreased in the lysates of breast cancer cell lines, and aberrant expression of the two proteins in breast cancer tissue was found (71). In recent studies, a proteomic analysis of 14 matched pairs of tumor tissues (ER-positive) prior to and following neoadjuvant treatment with aromatase inhibitor (AI) revealed 10 differentially expressed proteins. Among the identified proteins, heat shock protein 70 (HSP-70) was the most significantly correlated with both clinical and pathological responses (72). Subsequently, the downregulation of HSP-70 was assessed by IHC. Decrement of HSP-70 and Ki-67 following AI treatment and pretreatment were significantly associated with clinical responsiveness to $\mathrm{AI}$, demonstrating that HSP-70 is a potential predictive marker.

In addition, HSP-60 overexpression is correlated with the presence of axillary lymph node metastasis in breast cancers, indicating its potential prognostic value (73). Similarly, HSP-70 overexpression was correlated with a poor prognosis. This observation is consistent with the HSP-70 association with poor differentiation, lymph node metastasis, increased cell proliferation and blockage of apoptosis. Furthermore, the overexpression of HSP-90 and the presence of autoantibodies to HSP-90 have been correlated with a poor prognosis. HSP-70 is able to chaperone the oncoprotein HER-2/neu and the mutated $\mathrm{p} 53$, protecting these molecules from degradation by the proteasome, maintaining a less differentiated and more aggressive tumor phenotype (74). Further studies are required to determine the definitive value of the identified proteins as predictive markers. 


\section{Conclusions}

The majority of MS protein profiling studies exhibit promising diagnostic, prognostic or predictive values in breast cancer. Although it is indispensable for providing insight into the pathophysiological mechanisms associated with the development of absolute quantitative assays, few of these mass-to-charge ratios have been satisfactorily validated (75). Moreover, the candidate markers that have been identified constitute normal cell proteins or high abundant blood proteins involved in either coagulation or the acute phase response. Although their biological mechanism cannot be linked directly to tumor biochemistry, protein profiling studies have increased the knowledge of their molecular mechanisms involved in cancer through the identification of discriminative proteins generated exclusively by cancer cells.

The proteomic methods being used in recent years are evolving at an increasing rate. From studies examined by the code updating committee of the American Society of Clinical Oncology (ASCO), which regulates the use of tumor markers in breast cancer, a number of candidate proteins were identified (76). Serum, needle aspiration fluid (NAF), tumor tissue and intercellular fluid (TIF) were tested as proteomic indicators showing promise for clinical value. However, repeated prospective studies should be performed with well-defined larger sampling groups, in different populations and with varying analytical methods. In clinical trials, there are approximately 40 studies in progress relating to proteomics on breast cancer. However, none of the proteomic profiling techniques have been validated sufficiently for use in patient care (77).

Due to the high-throughput, simultaneous analysis of large numbers of proteins using proteomic techniques, there is a great chance of false discovery (45). Therefore, a second independent technique (for example, immunoblotting) is used to confirm the identification and expression change of individual putative biomarkers which have been suggested from proteomic studies. For immunoblotting, 1D-PAGE is used to separate each protein sample according to the MW, and the proteins are then transferred onto a nitrocellulose membrane. Densitometry can be used to normalize gel loading differences and compare the intensity of the bands produced by the primary antibody from each sample, producing a quantitation of the fold change in expression. Immunoblotting relies on the availability of a reliable specific primary antibody for the precise protein species identified by the proteomic analysis.

Since the proteomic methodologies described do not provide any information regarding the cellular localization of the putative protein biomarkers, they require further analysis using methods that can provide clinical validation, relying on the availability of a sufficient number of suitable clinical samples with the required clinical information. IHC is a lowthroughput technique that is used to validate the expression and localization of a protein of interest in whole sections both of formalin-fixed and paraffin-embedded (FFPE) clinical tissue samples on glass microscope slides. High-throughput IHC can be achieved following the production of a suitable TMA (78). For this process, cores of tissue are collected from hundreds of different donor FFPE samples and co-embedded into a single new recipient FFPE block. Each slide of the composite tissue section can then be simultaneously screened for expression of one test protein using IHC. For non-tissue based assays, an ELISA format, with an immobilized capture antibody, may be used to simultaneously screen protein samples from multiple clinical samples. Alternatively, MS-based assays, including multiple reaction monitoring (MRM) analysis, may be designed for the quantitation and validation of putative biomarkers in clinical samples. Following the successful validation and rigorous testing of putative biomarkers (79), a few may eventually find a role in clinical use.

In the definition of cancer indicators, proteomic technologies are producing valuable information, including differentiation, defining functional and regulatory pathways, determining the structure of disease-causing molecules in tissue and biologic fluids, and manifesting the disease stages or differences specific to the disease or individual. Since serum is generated by coagulation, its proteome is prone to the proteases involved in this cascade, as well as to those involved in the complement cascade activated upon clotting. Various pre-analytical parameters, including sampling device, clotting temperature and storage time, are all able to exert a distinct effect on the serum proteome. Thus, the concept of cancer type-specific protein fragments generated by tumor-secreted proteases awaits confirmation by validation studies that adhere to rigorous sample handling protocols.

From all of these data, it appears that the set of differentially expressed proteins identified from one study to another are variable, suggesting a lack of experimental standardization or issues of heterogeneity between the biological materials used in each study. Moreover, the differences of expression were only quantitative and few or none of the proteins found are exclusive in cancer versus normal breast tissues (80). The frequently identified proteins consist of normal cell proteins and high-abundant serum proteins involved in blood coagulation and the acute inflammatory response. Since the candidate proteins are among the least abundant, they may be below the detection threshold of the methods used. Therefore, it is likely that the specific proteins secreted by the tumor have yet to be detected (81).

Moreover, a number of the identified candidate breast cancer markers have been found to bear diagnostic potential for other cancer types; the majority of cancer types of epithelial origin share similar molecular features. Therefore, it may prove difficult to identify a true cancer-specific protein that is expressed exclusively by one type of malignant cell. Identification of specific tumor-secreted proteins is no prerequisite for improving breast cancer care, since improved breast cancer diagnosis, prognosis and prediction may also be accomplished using surrogate biomarkers of disease (82). A class of proteins currently recognised by the Breast Cancer Research Foundation for their surrogate biomarkers is the highly-abundant circulatory proteins. These fragments are generated by cancer type-specific protease activity, superimposed on the coagulation and complement degradation proteolytic pathways. In addition, these fragments also result from the proteases specifically expressed by malignant cells within the tumor microenvironment for tumor invasion and metastasis (83); they proteolytically process the acute phase proteins generated by the host response to the tumor.

The few validation studies currently available have all been retrospective. None of the candidate markers identi- 
fied at present have been investigated in larger, prospective, clinical settings. Therefore, none of the candidate biomarkers discussed in this review have been validated sufficiently to be used in clinical application (76). Translation from the discovery phase to the pre-clinical and subsequent clinical validation phase is required, as the sole purpose of a biomarker lies in its value. Nonetheless, the results of MALDI and SELDI-TOF MS protein profiling studies hold promise as high-throughput screening tools for the discovery of novel breast cancer markers. Provided that these studies are performed with adequate statistics and rigorous analysis, these protein biomarkers may improve cancer patient outcome.

In a systematic review of 20 MALDI/SELDI studies, Callesen et al (84) compared discriminatory peaks of candidates to diagnostic markers. These authors reported the occurrence of substantial heterogeneity in the studies with regards to experimental design, biological variation, pre-analytical conditions, collection and computational data analysis method. However, they still found common features among the studies, and demonstrated that $45 \%$ of the peaks previously related to breast cancer in these studies were also observed in a recent experimental study performed by the same authors. Studies testing the effects of different variables, including storage tubes, temperature, clotting time and incubation temperature, have shown that significant details for uniform handling to maintain systemic pre-analytical consistency are required (85). Non-standardized protocols in different validation studies have generated conflicting results, including clear variations in the discriminatory power and direction of several putative biomarkers.

Proteomics remains under development, and despite technical barriers that precede the use of proteomics analysis in clinical practice and the breast cancer complexity, MALDI-TOF and SELDI-TOF MS proteomic platforms with their innovations are powerful analytical tools for decoding alterations in the protein expression with respect to disease progression. These studies are required to be performed in a systematic manner with standardization of pre-analytical and analytical factors and computational tools for data treatment, in order that in the near future they are able to fulfil their role in personalized medicine and aid in the decrease of mortality from breast cancer.

\section{Acknowledgements}

This review was supported by the Shanghai Xuhui Science Foundation (No. xkkt201106).

\section{References}

1. Andersson T, Johansson M, Bolmsjo G and James P: Automating MALDI sample plate loading. J Proteome Res 6: 894-896, 2007.

2. Fink-Retter A, Czerwenka K, Gschwantler KD, et al: Proteomics in mammary cancer research. Eur J Gynaecol Oncol 30: 635-639, 2009.

3. Jemal A, Siegel R, Xu J and Ward E: Cancer statistics. CA Cancer J Clin 60: 277-300, 2010

4. Ries LA, Melbert D, Krapcho M, et al: SEER Cancer Statistics Review (http://seer.cancer.gov/csr/1975-2005). National Cancer Institute, 2008.

5. Bertucci F, Birnbaum D and Goncalves A: Proteomics of breast cancer: principles and potential clinical applications. Mol Cell Proteomics 5: 1772-1786, 2006.
6. Ma XJ, Dahiya S, Richardson E, et al: Gene expression profiling of the tumor microenvironment during breast cancer progression. Breast Cancer Res 11: R7, 2009.

7. Galva ERC, Martins LMS, Ibiapina JO, et al: Breast cancer proteomics: a review for clinicians. J Cancer Res Clin Oncol 137: 915-925, 2011.

8. Görg A, Drews O, Lück C, et al: 2-DE with IPGs. Electrophoresis 30 (Suppl 1): 122-132, 2009.

9. Baak JPA, Janssen EAM, Soreide K and Heikkilae R: Genomics and proteomics-the way forward. Ann Oncol 16 (Suppl 2): 30-34, 2005.

10. Bertucci F and Goncalves A: Clinical proteomics and breast cancer: strategies for diagnostic and therapeutic biomarker discovery. Future Oncol 4: 271-287, 2008.

11. Hamrita B, Nassr HB, Chahed K and Chouchane L: Proteomics approaches: new technologies and clinical applications in breast carcinomas. Gulf J Oncolog 1: 36-44, 2011.

12. Haab BB: Antibody arrays in cancer research. Mol Cell Proteomics 4: 377-383, 2005.

13. Nesvizhskii AI: Protein identification by tandem mass spectrometry and sequence database searching. Methods Mol Biol 367: 87-119, 2007.

14. Keller B, Sui J, Whittal YR, et al: Interferences and contaminants encountered in modern mass spectrometry. Anal Chim Acta 627: 71-81, 2008.

15. Yates JR, Ruse CI and Nakorchevsky A: Proteomics by mass spectrometry: approaches, advances and applications. Annu Rev Biomed Eng 11: 49-79, 2009.

16. Nesvizhskii AI, Vitek O and Aebersold R: Analysis and validation of proteomic data generated by tandem mass spectrometry. Nat Methods 4: 787-797, 2007.

17. Sutton CW, Rustogi N, Gurkan C, et al: Quantitative proteomic profiling of matched normal and tumor breast tissues. J Proteome Res 9: 3891-3902, 2010.

18. Bouchal P, Roumeliotis T, Hrstka R, et al: Biomarker discovery in low-grade breast cancer using isobaric stable isotope tags and two-dimensional liquid chromatography-tandem mass spectrometry (iTRAQ-2DLC-MS/MS) based quantitative proteomic analysis. J Proteome Res 8: 362-373, 2009.

19. Honrado E, Benitez J and Palacios J: Histopathology of BRCA1- and BRCA2-associated breast cancer. Crit Rev Oncol Hematal 59: 27-39, 2006.

20. Palacios J, Robles-Frias MJ, Castilla MA, et al: The molecular pathology of hereditary breast cancer. Pathobiology 75: 85-94, 2008.

21. Mangia A, Chiriatti A, Tommasi S, et al: BRCA1 expression and molecular alterations in familial breast cancer. Histol Histopathol 24: 69-76, 2009.

22. Reis-Filho JS and Tutt NA: Triple negative tumours: a critical review. Histopathology 52: 108-118, 2008.

23. Bhargava R, Beriwal S, McManus K and Dabbs DJ: CK5 is more sensitive than CK5/6 in identifying the 'basal-like' phenotype of breast carcinoma. Am J Clin Pathol 130: 724-730, 2008.

24. Korkola J and Gray JW: Breast cancer genomes-form and function. Curr Opin Genet Dev 20: 4-14, 2010.

25. Hudelist G, Singer CF, Pischinger KI, et al: Proteomic analysis in human breast cancer: identification of a characteristic protein expression profile of malignant breast epithelium. Proteomics 6: 1989-2002, 2006.

26. Pan S, Aebersold R, Chen R, et al: Mass spectrometry based targeted protein quantification: methods and applications. J Proteome Res 8: 787-797, 2009.

27. Braakman RB, Luider TM, Martens JW, et al: Laser capture microdissection applications in breast cancer proteomics. Methods Mol Biol 755: 143-154, 2011.

28. Umar A, Dalebou JC, Timmermans AM, et al: Method optimisation for peptide profiling of microdissected breast carcinoma tissue by matrix-assisted laser desorption/ionisation-time of flight and matrix-assisted laser desorption/ionisation-time of flight/time of flight-mass spectrometry. Proteomics 5: 2680-2688, 2005.

29. Sanders ME, Dias EC, Xu BJ, et al: Differentiating proteomic biomarkers in breast cancer by laser capture microdissection and MALDI MS. J Proteome Res 7: 1500-1507, 2008.

30. Orre LM, Pernemalm M, Lengqvist J, et al: Up-regulation, modification and translocation of S100A6 induced by exposure to ionizing radiation revealed by proteomics profiling. Mol Cell Proteomics 6: 2122-2131, 2007.

31. Moreira JM, Ohlsson G, Rank FE and Celis JE: Down-regulation of the tumor suppressor protein 14-3-3sigma is a sporadic event in cancer of the breast. Mol Cell Proteomics 4: 555-569, 2005. 
32. Zurita M, Lara PC, Moral RD, et al: Hypermethylated 14-3-3-o and ESR 1 gene promoters in serum as candidate biomarkers for the diagnosis and treatment efficacy of breast cancer metastasis. BMC Cancer 10: 217, 2010

33. Schultz J, Ibrahim SM, Vera J and Kunz M: 14-3-3o gene silencing during melanoma progression and its role in cell cycle control and cellular senescence. Mol Cancer 8: 53, 2009

34. Kabbage M, Chahed K, Hamrita B, et al: Protein alterations in infiltrating ductal carcinomas of the breast as detected by nonequilibrium $\mathrm{pH}$ gradient electrophoresis and mass spectrometry. J Biomed Biotechnol 2008: 564127, 2008.

35. Kamradt MC, Lu M, Werner ME, et al: The small heat shock protein alpha B-crystallin is a novel inhibitor of TRAIL-induced apoptosis that suppresses the activation of caspase-3. J Biol Chem 280: 11059-11066, 2005.

36. Moyano JV, Evans JR, Chen F, et al: Alpha B-crystallin is a nove oncoprotein that predicts poor clinical outcome in breast cancer. J Clin Invest 116: 261-270, 2006.

37. Li J, Orlandi R, White CN, et al: Independent validation of candidate breast cancer serum biomarkers identified by mass spectrometry. Clin Chem 51: 2229-2235, 2005.

38. Belluco C, Petricoin EF, Mammano E, et al: Serum proteomic analysis identifies a highly sensitive and specific discriminatory pattern in breast cancer. Ann Surg Oncol 14: 2470-2476, 2007.

39. Winden AWJ, Gast MCW, Beijnen JH, et al: Validation of previously identified serum biomarkers for breast cancer with SELDI-TOF MS: a case control study. BMC Med Genomics 2: 4, 2009 .

40. Winden AWJ, Broek I, Gast MCG, et al: Serum degradome markers for the detection of breast cancer. J Proteome Res 9 : 3781-3788, 2010

41. Villanueva J, Shaffer DR, Philip J, et al: Differential exoprotease activities confer tumor-specific serum peptidome patterns. J Clin Invest 116: 271-284, 2006.

42. Winden AW, Krop EJ, Karedal MH, et al: Searching for early breast cancer biomarkers by serum protein profiling of pre-diagnostic serum: a nested case-control study. BMC Cancer 11: 381 , 2011.

43. Pietrowska M, Polanska J, Marczak L, et al: Mass spectrometry-based analysis of therapy-related changes in serum proteome patterns of patients with early-stage breast cancer. J Transl Med 8: 66, 2010.

44. Schulz DM, Bollner C, Thomas G, et al: Identification of differentially expressed proteins in triple-negative breast carcinomas using DIGE and mass spectrometry. J Proteome Res 8 : 3430-3438, 2009.

45. Zakharchenko O, Greenwood C, Lewandowska A, et al: Meta-data analysis as a strategy to evaluate individual and common features of proteomic changes in breast cancer. Cancer Genomics Proteomics 8: 1-14, 2011.

46. Drake RR, Lisa H, Cazares LH, et al: Challenges to developing proteomic-based breast cancer diagnostics. OMICS 15: 251-259, 2011.

47. Foekens JA, Atkins D, Zhang Y, et al: Multicenter validation of a gene expression-based prognostic signature in lymph node-negative primary breast cancer. J Clin Oncol 24: 1665-1671, 2006.

48. Buyse M, Loi S, van't Veer L, et al: Validation and clinical utility of a 70-gene prognostic signature for women with node-negative breast cancer. J Natl Cancer Inst 98: 1183-1192, 2006.

49. Bueno-de-Mesquita JM, van Harten WH, Retel VP, et al: Use of 70-gene signature to predict prognosis of patients with node-negative breast cancer: a prospective community-based feasibility study (RASTER). Lancet Oncol 8: 1079-1087, 2007.

50. Desmedt C, Giobbie-Hurder A, Neven P, et al: The gene expression grade index: a potential predictor of relapse for endocrine-treated breast cancer patients in the BIG 1-98 trial. BMC Med Genomics 2: 40, 2009.

51. Gast MCW, Tinteren HV, Bontenbal M, et al: Haptoglobin phenotype is not a predictor of recurrence free survival in high-risk primary breast cancer patients. BMC Cancer 8: 389, 2008

52. Ricolleau G, Charbonnel C, Lode L, et al: Surface-enhanced laser desorption/ionization time of flight mass spectrometry protein profiling identifies ubiquitin and ferritin light chain as prognostic biomarkers in node-negative breast cancer tumors. Proteomics 6: 1963-1975, 2006.

53. Zeidan BA and Townsend PA: SELDI-TOF proteomic profiling of breast carcinomas identifies clinicopathologically relevant groups of patients similar to previously defined clusters from cDNA expression. Breast Cancer Res 10: 107, 2008
54. Baskin Y and Yigitbasi T: Clinical proteomics of breast cancer. Curr Genomics 11: 528-536, 2010.

55. Nakagawa T, Huang SK, Martinez SR, et al: Proteomic profiling of primary breast cancer predicts axillary lymph node metastasis. Cancer Res 66: 11825-11830, 2006.

56. Leong S, Christopherson RI and Baxter RC: Profiling of apoptotic changes in human breast cancer cells using SELDI-TOF mass spectrometry. Cell Physiol Biochem 20: 579-590, 2007.

57. Gast MC, van Dulken EJ, van Loenen TK, et al: Detection of breast cancer by surface-enhanced laser desorption/ionization time-of-flight mass spectrometry tissue and serum protein profiling. Int J Biol Markers 24: 130-141, 2009.

58. Brozkova K, Budinska E, Bouchal P, et al: Surface-enhanced laser desorption/ionization time-of-flight proteomic profiling of breast carcinomas identifies clinicopathologically relevant groups of patients similar to previously defined clusters from cDNA expression. Breast Cancer Res 10: R48, 2008.

59. Goncalves A, Charafe-Jauffret E, Bertucci F, et al: Protein profiling of human breast tumor cells identifies novel biomarkers associated with molecular subtypes. Mol Cell Proteomics 7: 1420-1433, 2008

60. Traub F, Jost M, Hess R, et al: Peptidomic analysis of breast cancer reveals a putative surrogate marker for estrogen receptor-negative carcinomas. Lab Invest 86: 246-253, 2006.

61. Harigopal M, Heymann J, Ghosh S, et al: Estrogen receptor co-activator (AIB1) protein expression by automated quantitative analysis (AQUA) in a breast cancer tissue microarray and association with patient outcome. Breast Cancer Res Treat 115: 77-85, 2009.

62. Hodgkinson VC, Eagle GL, Drew PJ, et al: Biomarkers of chemotherapy resistance in breast cancer identified by proteomics: current status. Cancer Lett 294: 13-24, 2010.

63. Villeneuve DJ, Hembruff SL, Veitch Z, et al: cDNA microarray analysis of isogenic paclitaxel- and doxorubicin-resistant breast tumor cell lines reveals distinct drug-specific genetic signatures of resistance. Breast Cancer Res Treat 96: 17-39, 2006

64. Maurya P, Meleady P, Dowling P and Clynes M: Proteomic approaches for serum biomarker discovery in cancer. Anticancer Res 27: 1247-1255, 2007.

65. Nakayama S, Torikoshi Y, Takahashi T, et al: Prediction of paclitaxel sensitivity by CDK1 and CDK 2 activity in human breast cancer cells. Breast Cancer Res 11: R12, 2009.

66. Riley CP, Zhang X, Nakshatei H, et al: A large, consistent plasma proteomics data set from prospectively collected breast cancer patient and healthy volunteer samples. J Transl Med 9: 80, 2011.

67. Kulasingam V and Diamandis EP: Proteomics analysis of conditioned media from three breast cancer cell lines: a mine for biomarkers and therapeutic targets. Mol Cell Proteomics 6: 1997-2011, 2007

68. Loi S, Haibe-Kains B, Majjaj S, et al: PIK3CA mutations associated with gene signature of low mTORC1 signaling and better outcomes in estrogen receptor-positive breast cancer. Proc Natl Acad Sci USA 107: 10208-10213, 2010

69. Colombo PE, Milanezi F, Weigelt B and Reis-Filho JS: Microarrays in the 2010s: the contribution of microarray-based gene expression profiling to breast cancer classification, prognostication and prediction. Breast Cancer Res 13: 212, 2011.

70. Liu Y, Liu H, Han B and Zhang JT: Identification of 14-3-3 $\sigma$ as a contributor to drug resistance in human breast cancer cells using functional proteomic analysis. Cancer Res 66: 3248, 2006.

71. Gast MC, Schellens JH and Beijnen JH: Clinical proteomics in breast cancer: a review. Breast Cancer Res Treat 116: 17-29, 2009.

72. Yiu CC, Chanplakorn N, Chan MS, et al: Down regulation of heat shock protein 70 (HSP-70) correlated with responsiveness to neoadjuvant aromatase inhibitor (AI) therapy in breast cancer patients. Anticancer Res 30: 3465-3472, 2010.

73. You BC, Park SP, Lee YD, et al: The expression of heat shock protein $60 \mathrm{kDa}$ in tissues and cell lines of breast cancer. Breast Cancer 11: 161-171, 2008

74. Zagouri F, Sergentanis TN, Nonni A, et al: Hsp90 in the continuum of breast ductal carcinogenesis: evaluation in precursors, preinvasive and ductal carcinoma lesions. BMC Cancer 10: 353, 2010.

75. Goncalves A and Bertucci F: Clinical application of proteomics in breast cancer: state of the art and perspectives. Med Princ Prac 20: 4-18, 2011.

76. Haris L, Fritsche H, Mannel R, et al: American Society of Clinical Oncology 2007 update of recommendations for the use of tumor markers in breast cancer. J Clin Oncol 25: 1-26, 2007.

77. Makridakis M and Vlahou A: Secretome proteomics for discovery of cancer biomarkers. J Proteomics 73: 2291-2305, 2010 
78. Camp RL, Neumeister V, Rimm DL, et al: A decade of tissue microarrays: progress in the discovery and validation of cancer biomarkers. J Clin Oncol 26: 5630-5637, 2008.

79. Brennan DJ, Kelly C, Rexhepaj E, et al: Contribution of DNA and tissue microarray technology to the identification and validation of biomarkers and personalised medicine in breast cancer. Cancer Genomics Proteomic 4: 121-134, 2007.

80. Hondermarck H, Tastet C, Yazidi-Belkoura El, et al: Proteomics of breast cancer: the quest for markers and therapeutic targets. J Proteome Res 7: 1403-1411, 2008.

81. Gilabert M, Audebert S, Viens P, et al: Proteomics and breast cancer: a search for novel diagnostic and theragnostic biomarkers. Bull Cancer 97: 321-339, 2010.

82. Rodenhiser DI, Andrews JD, Vandenberg TA and Chambers AF: Gene signatures of breast cancer progression and metastasis. Breast Cancer Res 13: 201, 2011.
83. Tuck MK, Chan DW, Chia D, et al: Standard operating procedures for serum and plasma collection:early detection research net work consensus statement standard operating procedure integration working group. J Proteome Res 8: 113-117, 2009.

84. Callesen AK, Vach W, Jorgensen PE, et al: Reproducibility of mass spectrometry based protein profiles for diagnosis of breast cancer across clinical studies: a systematic review. J Proteome Res 7: 1395-1402, 2008

85. Zeidan BA, Cutress RI, Murray N, et al: Proteomic analysis of archival breast cancer serum. Cancer Genomics Proteomics 6: 141-147, 2009. 